\title{
ON ALGEBRAIC PROPERTIES AND LINEARITY OF OWA OPERATORS FOR FUZZY SETS
}

\author{
ZDENKO TAKÁČ
}

\begin{abstract}
We deal with an ordered weighted averaging operator (OWA operator) on the set of all fuzzy sets. Our starting point is OWA operator on any lattice introduced in Lizasoain, I.-Moreno, C.: OWA operators defined on complete lattices, Fuzzy Sets and Systems 224 (2013), 36-52; Ochoa, G.--Lizasoain, I.-Paternain, D.-Bustince, H.-Pal, N. R.: Some properties of lattice OWA operators and their importance in image processing, in: Proc. of the 16th World Congress of the Internat. Systems Assoc.-IFSA '15 and the 9th Conf. of the European Soc. for Fuzzy Logic and Technology-EUSFLAT '15 (J. M. Alonso et al., eds.), Atlantis Press, Gijón, Spain, 2015, pp. 1261-1265. We focus on a particular case of lattice, namely that of all normal convex fuzzy sets in $[0,1]$, and study algebraic properties and linearity of the proposed OWA operator. It is shown that the operator is an extension of standard OWA operator for real numbers and it possesses similar algebraic properties as standard one, however, it is neither homogeneous nor shift-invariant, i.e., it is not linear in contrast to the standard OWA operator.
\end{abstract}

\section{Introduction}

The aggregation of fuzzy sets, that is fuzzy truth values, is essential in the type-2 fuzzy sets settings [10], [11. Also, the need of aggregation of fuzzy sets arises in decision making problems when the alternatives are assessed by fuzzy sets. Recall that Yager's OWA operators are of special significance in solving decision making problems. This leads to growing interest of scholars to investigate OWA operators for various kinds of elements [4], e.g., for intervals [2], 15], fuzzy intervals [17], [18, gradual intervals [12, i.e., also for fuzzy sets.

In [6], 8] the concept of an ordered weighted averaging (OWA for short) operator is extended to any complete lattice endowed with a t-norm and a t-conorm. The intention of authors was to avoid the need of a linear order in environments

(C) 2016 Mathematical Institute, Slovak Academy of Sciences. 2010 Mathematics Subject Classification: 03E72.

Keywords: OWA operator, type-2 fuzzy set, fuzzy set, fuzzy truth value, aggregation, distributive weighting vector.

This work was supported by the Grant VEGA 1/0420/15. 


\section{ZDENKO TAKÁČ}

in which only partial, non-linear, order is available. Our aim is to study a specific nature of ideas from [6], [8] in one particular case of complete lattice, namely that of all normal convex fuzzy truth values (fuzzy sets in $[0,1]$ ). It is well-known that this set is not linearly ordered. We discuss the notion of (distributive) weighting vector, formulate a sufficient and necessary condition under which given elements constitute a distributive weighting vector and study algebraic properties and linearity of the proposed OWA operator for fuzzy sets.

The paper is organized as follows. Section 2 contains basic definitions and notations that are used in the remaining parts of the paper. In Section 3, we propose an OWA operator on the set of normal convex fuzzy sets in $[0,1]$ and study its properties. The conclusions are discussed in Section 4.

\section{Preliminaries}

In this section, we present some basic concepts and terminology that will be used throughout the paper.

Let $X$ be a set. A fuzzy set in $X$ is a mapping from $X$ to $[0,1]$. Let $\mathcal{F}(X)$ denote the class of all fuzzy sets in $X$, and let $\mathcal{F}$ denote the class of all fuzzy sets in $[0,1]$. A type-2 fuzzy set in $X$ is a fuzzy set whose membership grades are fuzzy sets in $[0,1]$. Hence, type-2 fuzzy set in $X$ is a mapping

$$
\tilde{f}: X \rightarrow \mathcal{F}
$$

and the elements of $\mathcal{F}$ are called fuzzy truth values.

A fuzzy set $f$ in $X$ is normal if there exists $x \in X$ such that $f(x)=1$. Let $X$ be a linear space, a fuzzy set $f$ in $X$ is convex if it satisfies $f\left(\lambda x_{1}+(1-\lambda) x_{2}\right) \geq$ $\min \left(f\left(x_{1}\right), f\left(x_{2}\right)\right)$ for all $\lambda \in[0,1]$, for each $x_{1}, x_{2} \in X$. We denote by $\mathcal{F}_{N C}$ the class of all normal convex fuzzy truth values. We will use operations $\sqcup, \sqcap$, relations $\sqsubseteq, \preceq$ and special elements $\widetilde{0}, \widetilde{1}$ on $\mathcal{F}$ given by:

$$
\begin{aligned}
& (f \sqcup g)(z)=\sup _{x \vee y=z}(f(x) \wedge g(y)), \quad f \sqsubseteq g \text { if and only if } f \sqcap g=f, \\
& (f \sqcap g)(z)=\sup _{x \wedge y=z}(f(x) \wedge g(y)), \quad f \preceq g \quad \text { if and only if } \quad f \sqcup g=g \text {, } \\
& \widetilde{0}(x)=\left\{\begin{array}{ll}
1 & \text { if } x=0, \\
0, & \text { otherwise, }
\end{array} \quad \widetilde{1}(x)= \begin{cases}1 & \text { if } x=1, \\
0, & \text { otherwise. }\end{cases} \right.
\end{aligned}
$$

The algebra of fuzzy truth values $(\mathcal{F}, \sqcup, \sqcap, \widetilde{0}, \underset{1}{\widetilde{1}}, \sqsubseteq, \preceq)$ is closely described in $[7]$ and [13]. In [13] it is showed that $\left(\mathcal{F}_{N C}, \sqcup, \sqcap, \widetilde{0}, \widetilde{1}, \sqsubseteq\right)$ is a bounded, distributive lattice, and in [3] the authors showed that the lattice is complete. Recall that the two orders $\sqsubseteq$ and $\preceq$ coincide on the set of normal convex fuzzy sets.

In $1988 \mathrm{Yager}$ [16] introduced OWA operator which is one of the most widely used aggregation methods for real numbers. 
ON ALGEBRAIC PROPERTIES AND LINEARITY OF OWA OPERATORS...

Definition 2.1. Let $\mathbf{w}=\left(w_{1}, \ldots, w_{n}\right) \in[0,1]^{n}$ be a weighting vector with $w_{1}+\cdots+w_{n}=1$. An OWA operator associated with $\mathbf{w}$ is a mapping $O W A_{\mathrm{w}}:[0,1]^{n} \rightarrow[0,1]$ defined by

$$
O W A_{\mathbf{w}}\left(x_{1}, \ldots, x_{n}\right)=\sum_{i=1}^{n} w_{i} x_{(i)},
$$

where $x_{(i)}$ denotes the $i$ th largest number among $x_{1}, \ldots, x_{n}$.

\section{OWA operators defined on the set of all normal convex fuzzy sets in $[0,1]$}

In this section we apply the ideas of $[6,8$ to the settings of type-2 fuzzy sets. In other words, we will study distributive weighting vectors and consequently OWA operators on the set of fuzzy truth values $\mathcal{F}$. Let us start with the notion of a t-norm and a t-conorm on $\mathcal{F}$.

Definition 3.1. A mapping $T: \mathcal{F} \times \mathcal{F} \rightarrow \mathcal{F}$ is said to be a t-norm on $(\mathcal{F}, \sqsubseteq)$ if it is commutative, associative, increasing in each component and has a neutral element $\widetilde{1}$.

A mapping $S: \mathcal{F} \times \mathcal{F} \rightarrow \mathcal{F}$ is said to be a t-conorm on $(\mathcal{F}, \sqsubseteq)$ if it is commutative, associative, increasing in each component and has a neutral element $\widetilde{0}$.

The operations $\sqcap$ and $\sqcup$ given by (11) are t-norm and t-conorm on $\mathcal{F}$, respectively. The following propositions are easy to check, see [5], 9] and [14].

Proposition 3.2. The operation $\sqcap$ given by (11) is a $t$-norm on $(\mathcal{F}, \sqsubseteq)$.

Proposition 3.3. The operation $\sqcup$ given by (11) is a $t$-conorm on $(\mathcal{F}, \sqsubseteq)$.

According to the following lemma, it is possible to construct linearly ordered vector from any given vector in $\mathcal{F}_{N C}^{n}$.

Lemma $3.4([6])$. Let $\left(f_{1}, \ldots, f_{n}\right) \in \mathcal{F}_{N C}^{n}$, and let

$$
\begin{aligned}
& g_{1}=f_{1} \sqcup \ldots \sqcup f_{n}, \\
& g_{2}=\left(\left(f_{1} \sqcap f_{2}\right) \sqcup \ldots \sqcup\left(f_{1} \sqcap f_{n}\right)\right) \sqcup\left(\left(f_{2} \sqcap f_{3}\right) \sqcup \ldots \sqcup\left(f_{2} \sqcap f_{n}\right)\right) \sqcup \ldots \sqcup\left(\left(f_{n-1} \sqcap f_{n}\right)\right), \\
& \quad \vdots \\
& g_{k}=\sqcup\left\{f_{j_{1}} \sqcap \ldots \sqcap f_{j_{k}} \mid\left\{j_{1}, \ldots, j_{k}\right\} \subseteq\{1, \ldots, n\}\right\}, \\
& \quad \vdots \\
& g_{n}=f_{1} \sqcap \ldots \sqcap f_{n} .
\end{aligned}
$$

Then

$$
g_{n} \sqsubseteq g_{n-1} \sqsubseteq \ldots \sqsubseteq g_{1} .
$$

Moreover, if the set $\left\{f_{1}, \ldots, f_{n}\right\}$ is linearly ordered, then the vector $\left(g_{1}, \ldots, g_{n}\right)$ coincides with $\left(f_{\sigma(1)}, \ldots, f_{\sigma(n)}\right)$ for some permutation $\sigma$ of $\{1, \ldots, n\}$. 


\section{ZDENKO TAKÁČ}

We proceed with the study of distributive weighting vector in $\mathcal{F}_{N C}^{n}$ and some of its properties.

Definition 3.5. Let $w_{1}, \ldots, w_{n} \in \mathcal{F}$. A vector $\left(w_{1}, \ldots, w_{n}\right)$ is said to be a weighting vector in $(\mathcal{F}, \sqsubseteq)$ if $w_{1} \sqcup \ldots \sqcup w_{n}=\widetilde{1}$, and it is said to be a distributive weighting vector if it also satisfies

$$
f \sqcap\left(w_{1} \sqcup \ldots \sqcup w_{n}\right)=\left(f \sqcap w_{1}\right) \sqcup \ldots \sqcup\left(f \sqcap w_{n}\right)
$$

for all $f \in \mathcal{F}$.

The following theorem gives a necessary condition under which elements $w_{1}, \ldots, w_{n}$ constitute a weighting vector in $\left(\mathcal{F}_{N C}, \sqsubseteq\right)$.

TheOREM 3.6. Let $w_{1}, \ldots, w_{n} \in \mathcal{F}_{N C}$. If $w_{1} \sqcup \ldots \sqcup w_{n}=\widetilde{1}$, then $w_{i}=\widetilde{1}$ for some $i \in\{1, \ldots, n\}$.

Proof.

1. We show that $w_{i}(1)=1$ for some $i \in\{1, \ldots, n\}$. From $w_{1} \sqcup \ldots \sqcup w_{n}=\widetilde{1}$ it follows $\left(w_{1} \sqcup \ldots \sqcup w_{n}\right)(1)=1$, hence there exist $a_{1}, \ldots a_{n} \in[0,1]$ such that $\max \left(a_{1}, \ldots, a_{n}\right)=1$ and $\min \left(w_{1}\left(a_{1}\right), \ldots, w_{n}\left(a_{n}\right)\right)=1$, and consequently there exist $a_{1}, \ldots a_{n} \in[0,1]$ such that $\max \left(a_{1}, \ldots, a_{n}\right)=1$ and $w_{1}\left(a_{1}\right)=\ldots=$ $w_{n}\left(a_{n}\right)=1$. It means that for some $i \in\{1, \ldots, n\}$ it holds $a_{i}=1$ and $w_{i}\left(a_{1}\right)=1$, thus $w_{i}(1)=1$ for some $i \in\{1, \ldots, n\}$. Let us write $w_{k_{0}}(1)=1$.

2. Now we are going to show that $w_{k_{0}}(x)=0$ for all $x \in\left[0,1\left[\right.\right.$ if $w_{i} \neq \tilde{1}$ for all $i \in\{1, \ldots, n\}-\left\{k_{0}\right\}$. Let there exist $x_{0} \in\left[0,1\left[\right.\right.$ such that $w_{k_{0}}\left(x_{0}\right)>0$. Then there exist

$b_{1}, \ldots, b_{k_{0}-1}, b_{k_{0}+1}, \ldots, b_{n} \in[0,1[$ such that

$$
w_{1}\left(b_{1}\right), \ldots, w_{k_{0}-1}\left(b_{k_{0}-1}\right), w_{k_{0}+1}\left(b_{k_{0}+1}\right), \ldots, w_{n}\left(b_{n}\right)>0,
$$

hence

$$
\begin{aligned}
& \left(w_{1} \sqcup \ldots \sqcup w_{n}\right)\left(\max \left(b_{1}, \ldots, b_{k_{0}-1}, x_{0}, b_{k_{0}+1}, \ldots, b_{n}\right)\right) \\
& =\min \left(w_{1}\left(b_{1}\right), \ldots, w_{k_{0}-1}\left(b_{k_{0}-1}\right), w_{k_{0}}\left(x_{0}\right), w_{k_{0}+1}\left(b_{k_{0}+1}\right), \ldots, w_{n}\left(b_{n}\right)\right)>0,
\end{aligned}
$$

which contradicts our assumption $w_{1} \sqcup \ldots \sqcup w_{n}=\widetilde{1}$.

The following corollary states a simple necessary and sufficient condition under which $\left(w_{1}, \ldots, w_{n}\right) \in \mathcal{F}_{N C}^{n}$ is a distributive weighting vector.

CoRollaRY 3.7. A vector $\left(w_{1}, \ldots, w_{n}\right) \in \mathcal{F}_{N C}^{n}$ is a distributive weighting vector in $\left(\mathcal{F}_{N C}, \sqsubseteq\right)$ if and only if there exists $i \in\{1, \ldots, n\}$ such that $w_{i}=\widetilde{1}$.

Proof.

1. Necessity: Let $\left(w_{1}, \ldots, w_{n}\right) \in \mathcal{F}_{N C}^{n}$ be a distributive weighting vector. Then, according to Definition 3.5, $w_{1} \sqcup \ldots \sqcup w_{n}=\widetilde{1}$; and from Theorem 3.6 it follows $w_{i}=\widetilde{1}$ for some $i \in\{1, \ldots, n\}$. 
2. Sufficiency: Let us first observe that $\left(\mathcal{F}_{N C}, \sqsubseteq\right)$ is a distributive lattice, thus it is sufficient to show that $\left(w_{1}, \ldots, w_{n}\right)$ is a weighting vector in $\left(\mathcal{F}_{N C}, \sqsubseteq\right)$. Let $w_{i}=\widetilde{1}$ for some $i \in\{1, \ldots, n\}$. The proof follows from the observation that $\widetilde{1} \sqcup f=\widetilde{1}$ for all $f \in \mathcal{F}_{N C}$.

Now we can use the notion of distributive weighting vector and define an OWA operator on the set of normal convex fuzzy truth values $\mathcal{F}_{N C}$.

Definition 3.8. Let $\mathbf{w}=\left(w_{1}, \ldots, w_{n}\right) \in \mathcal{F}_{N C}^{n}$ be a distributive weighting vector in $\left(\mathcal{F}_{N C}, \sqsubseteq\right)$. The mapping $F_{\mathbf{w}}: \mathcal{F}_{N C}^{n} \rightarrow \mathcal{F}_{N C}$ given, for all $\left(f_{1}, \ldots, f_{n}\right) \in$ $\mathcal{F}_{N C}^{n}$, by

$$
F_{\mathbf{w}}\left(f_{1}, \ldots, f_{n}\right)=\left(w_{1} \sqcap g_{1}\right) \sqcup \ldots \sqcup\left(w_{n} \sqcap g_{n}\right),
$$

where $\left(g_{1}, \ldots, g_{n}\right)$ is a linearly ordered vector constructed from $\left(f_{1}, \ldots, f_{n}\right)$ according to Lemma 3.4 , is called an $n$-ary OWA operator on $\mathcal{F}_{N C}$.

EXAMPLE 3.9. Let weighting vector be $\mathbf{w}=\left(w_{1}, \widetilde{1}\right)$ and $w_{1}, f_{1}, f_{2}$ be fuzzy truth values given by Fig. 11. Then $g_{1}=f_{1} \sqcup f_{2}, g_{2}=f_{1} \sqcap f_{2}$, and

$$
F_{\mathbf{w}}\left(f_{1}, f_{2}\right)=\left(w_{1} \sqcap g_{1}\right) \sqcup\left(\widetilde{1} \sqcap g_{2}\right)=\left(w_{1} \sqcap g_{1}\right) \sqcup g_{2} .
$$

The results are depicted in Fig. 11 (for simplicity, fuzzy truth values $g_{1}$ and $g_{2}$ are not depicted - they can be found in Fig. 2).

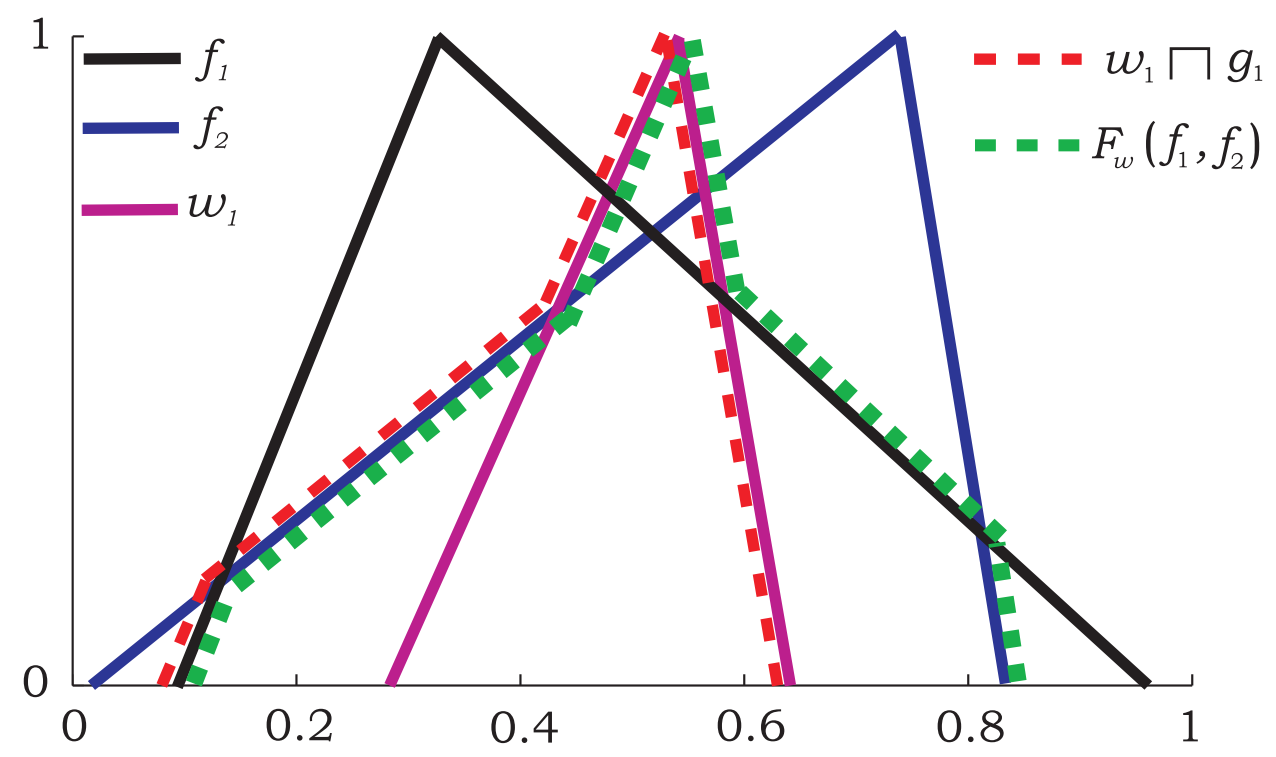

Figure 1. See Example 3.9 


\section{ZDENKO TAKÁČ}

The remainder of this paper will be devoted to the study of properties of the $n$-ary OWA operator on $\mathcal{F}_{N C}$. In the following theorem we show that the operator satisfies three basic properties of each aggregation operator, i.e., it is aggregation operator on $\mathcal{F}_{N C}$.

TheOREM 3.10. Let $\mathbf{w}=\left(w_{1}, \ldots, w_{n}\right)$ be a distributive weighting vector in $\left(\mathcal{F}_{N C}, \sqcap, \sqcup, \sqsubseteq, \widetilde{0}, \widetilde{1}\right)$ and $F_{\mathbf{w}}$ the corresponding OWA operator given by Definition 3.8. Then:

(i) $F_{\mathbf{w}}(\widetilde{0}, \ldots, \widetilde{0})=\widetilde{0}$.

(ii) $F_{\mathbf{w}}(\widetilde{1}, \ldots, \widetilde{1})=\widetilde{1}$.

(iii) $f_{1} \sqsubseteq f_{1}^{*}, \ldots, f_{n} \sqsubseteq f_{n}^{*}$ imply

$F_{\mathbf{w}}\left(f_{1}, \ldots, f_{n}\right) \sqsubseteq F_{\mathbf{w}}\left(f_{1}^{*}, \ldots, f_{n}^{*}\right) \quad$ for all $f_{1}, f_{1}^{*}, \ldots, f_{n}, f_{n}^{*} \in \mathcal{F}_{N C}$.

Proof.

(i) $F_{\mathbf{w}}(\widetilde{0}, \ldots, \widetilde{0})=\left(w_{1} \sqcap \widetilde{0}\right) \sqcup \ldots \sqcup\left(w_{n} \sqcap \widetilde{0}\right)=\widetilde{0} \sqcup \ldots \sqcup \widetilde{0}=\widetilde{0}$.

(ii) $F_{\mathbf{w}}(\widetilde{1}, \ldots, \widetilde{1})=\left(w_{1} \sqcap \widetilde{1}\right) \sqcup \ldots \sqcup\left(w_{n} \sqcap \widetilde{1}\right)=w_{1} \sqcup \ldots \sqcup w_{n}=\widetilde{1}$.

(iii) Let $f_{1} \sqsubseteq f_{1}^{*}, \ldots, f_{n} \sqsubseteq f_{n}^{*}$. Then we have

$$
g_{1} \sqsubseteq g_{1}^{*}, \ldots, g_{n} \sqsubseteq g_{n}^{*}
$$

and consequently

$$
\begin{aligned}
& F_{\mathbf{w}}\left(f_{1}, \ldots, f_{n}\right) \\
& =\left(w_{1} \sqcap g_{1}\right) \sqcup \ldots \sqcup\left(w_{n} \sqcap g_{n}\right) \sqsubseteq\left(w_{1} \sqcap g_{1}^{*}\right) \sqcup \ldots \sqcup\left(w_{n} \sqcap g_{n}^{*}\right) \\
& =F_{\mathbf{w}}\left(f_{1}^{*}, \ldots, f_{n}^{*}\right) .
\end{aligned}
$$

TheORem 3.11. Let $F_{\mathbf{w}}$ be an n-ary $O W A$ operator on $\mathcal{F}_{N C}$. Then

$$
f_{1} \sqcap \ldots \sqcap f_{n} \sqsubseteq F_{\mathbf{w}}\left(f_{1}, \ldots, f_{n}\right) \sqsubseteq f_{1} \sqcup \ldots \sqcup f_{n} \quad \text { for all } f_{1}, \ldots, f_{n} \in \mathcal{F}_{N C} .
$$

P r o of. We prove the right inequality, the left one can be checked in a similar way.

$$
\begin{aligned}
F_{\mathbf{w}}\left(f_{1}, \ldots, f_{n}\right) & =\left(w_{1} \sqcap g_{1}\right) \sqcup \ldots \sqcup\left(w_{n} \sqcap g_{n}\right) \sqsubseteq\left(w_{1} \sqcap g_{1}\right) \sqcup \ldots \sqcup\left(w_{n} \sqcap g_{1}\right) \\
& =\left(w_{1} \sqcup \ldots \sqcup w_{n}\right) \sqcap g_{1}=\widetilde{1} \sqcap g_{1}=g_{1}=f_{1} \sqcup \ldots \sqcup f_{n} .
\end{aligned}
$$

The theorem says that the results of $F_{\mathbf{w}}\left(f_{1}, \ldots, f_{n}\right)$ are bounded by $f_{1} \sqcap \ldots \sqcap f_{n}$ and $f_{1} \sqcup \ldots \sqcup f_{n}$. It is worth pointing out that for standard OWA operators for real numbers from $\min \left(x_{1}, \ldots, x_{n}\right) \leq O W A_{w}\left(x_{1}, \ldots, x_{n}\right) \leq \max \left(x_{1}, \ldots, x_{n}\right)$ it follows that $O W A_{w}\left(x_{1}, \ldots, x_{n}\right) \geq x_{i}$ for some $i \in\{1, \ldots, n\}$, and $O W A_{w}\left(x_{1}, \ldots, x_{n}\right) \leq x_{j}$ 
ON ALGEBRAIC PROPERTIES AND LINEARITY OF OWA OPERATORS...

for some $j \in\{1, \ldots, n\}$. However, the similar property does not hold for $F_{\mathbf{w}}$, i.e., it is possible that

$$
F_{\mathbf{w}}\left(f_{1}, \ldots, f_{n}\right) \sqsubset f_{i} \quad \text { for all } i \in\{1, \ldots, n\}
$$

or

$$
F_{\mathbf{w}}\left(f_{1}, \ldots, f_{n}\right) \sqsupset f_{j} \quad \text { for all } j \in\{1, \ldots, n\} .
$$

See Example 3.12 where $F_{\mathbf{w}}\left(f_{1}, f_{2}\right) \sqsupset f_{1}$ and $F_{\mathbf{w}}\left(f_{1}, f_{2}\right) \sqsupset f_{2}$.

EXAMPLE 3.12. Let weighting vector be $\mathbf{w}=\left(\widetilde{1}, w_{2}\right)$ and $w_{2}, f_{1}, f_{2}$ be fuzzy truth values given by Fig. 2. Then $g_{1}=f_{1} \sqcup f_{2}, g_{2}=f_{1} \sqcap f_{2}$, and (see Lemma 3.13 for the last equality)

$$
F_{\mathbf{w}}\left(f_{1}, f_{2}\right)=\left(\widetilde{1} \sqcap g_{1}\right) \sqcup\left(w_{2} \sqcap g_{2}\right)=g_{1} \sqcup\left(w_{2} \sqcap g_{2}\right)=g_{1} .
$$

It is easy to check that $F_{\mathbf{w}}\left(f_{1}, f_{2}\right)=g_{1} \sqsupset f_{i}$, for $i=1,2$.

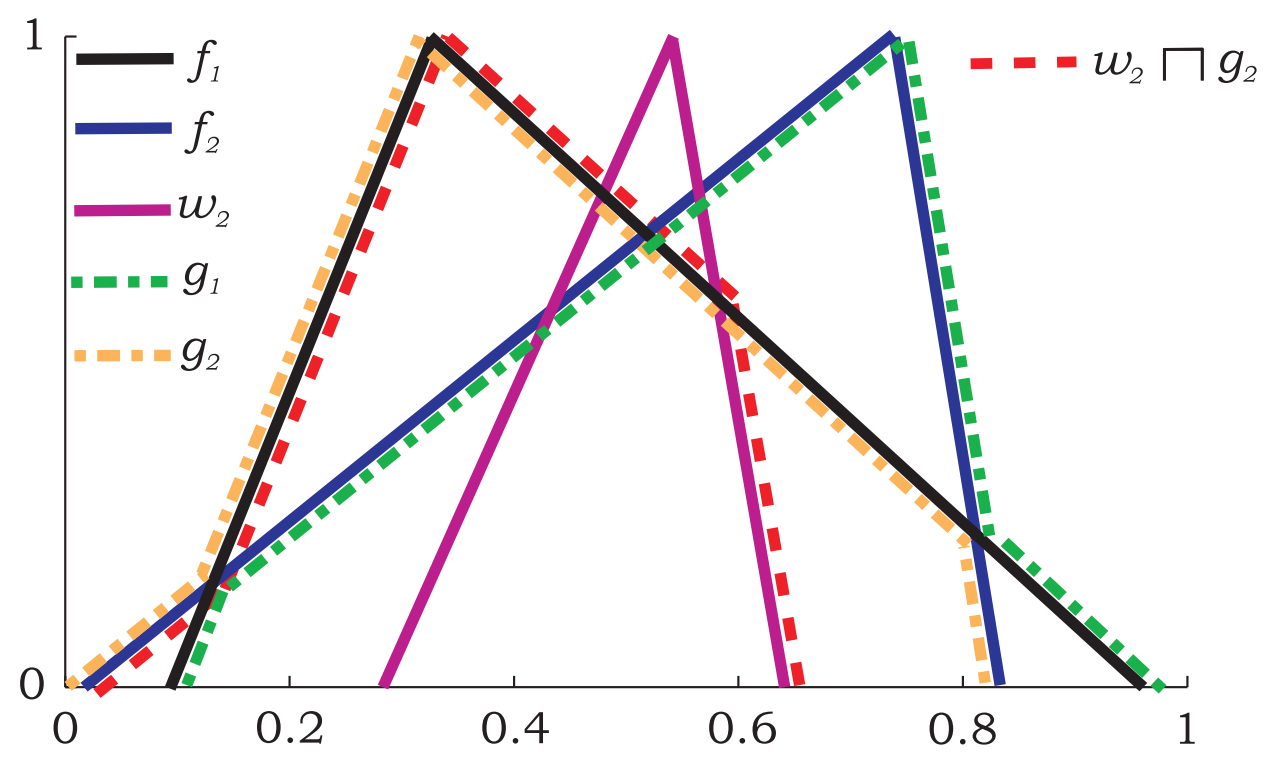

Figure 2. See Example 3.12

The following lemma was used in previous example and will also be needed in proof of Theorem 3.14 ,

LemMA 3.13. Let $f, g, h \in \mathcal{F}_{N C}$ with $f \sqsubseteq g$. Then $g \sqcup(f \sqcap h)=g$.

P r o of. Applying the distributive laws ([13, Proposition 36]) and absorption laws ([13, Proposition 37]) we have:

$$
g \sqcup(f \sqcap h)=(g \sqcup f) \sqcap(g \sqcup h)=g \sqcap(g \sqcup h)=g .
$$




\section{ZDENKO TAKÁČ}

Note that the property of Lemma 3.13 does not hold in $\mathcal{F}$. The reason is that the absorption laws fail if $g$ is not convex or $h$ is not normal.

We can now strengthen [6, Proposition 3.8] in the settings of fuzzy truth values. Our result is that if $\widetilde{1}$ is on the first position of a weighting vector $\mathbf{w}$, then our OWA operator is simply maximum, no matter what are the other weights - see item 1 of the following theorem. Note that there are much stronger assumptions for a similar assertion on minimum - see item 2 of the theorem.

Theorem 3.14. Let $\mathbf{w}=\left(w_{1}, \ldots, w_{n}\right)$ be a distributive weighting vector in $\mathcal{F}_{N C}$, and let $F_{\mathbf{w}}$ be an n-ary $O W A$ operator on $\mathcal{F}_{N C}$.

(i) If $w_{1}=\widetilde{1}$, then $F_{\mathbf{w}}\left(f_{1}, \ldots, f_{n}\right)=f_{1} \sqcup \ldots \sqcup f_{n}$.

(ii) If $w_{n}=\widetilde{1}$ and $w_{i} \sqsubseteq f_{1} \sqcap \ldots \sqcap f_{n}$ for all $i \in\{1, \ldots, n-1\}$, then $F_{\mathbf{w}}\left(f_{1}, \ldots, f_{n}\right)=f_{1} \sqcap \ldots \sqcap f_{n}$.

(iii) If $w_{k}=\widetilde{1}$ for some $k \in\{1, \ldots, n\}$ and $w_{i} \sqsubseteq g_{k}$ for all $i \in\{1, \ldots, k-1\}$, then $F_{\mathbf{w}}\left(f_{1}, \ldots, f_{n}\right)=g_{k}$.

Proof.

(i) Let $\mathbf{w}=\left(\widetilde{1}, w_{2}, \ldots, w_{n}\right)$. Then

$$
\begin{aligned}
F_{\mathbf{w}}\left(f_{1}, \ldots, f_{n}\right) & =\left(\widetilde{1} \sqcap g_{1}\right) \sqcup\left(w_{2} \sqcap g_{2}\right) \sqcup \ldots \sqcup\left(w_{n} \sqcap g_{n}\right) \\
& =g_{1} \sqcup\left(w_{2} \sqcap g_{2}\right) \sqcup \ldots \sqcup\left(w_{n} \sqcap g_{n}\right)
\end{aligned}
$$

and applying Lemma $3.13(n-1)$ times we conclude

$$
F_{\mathbf{w}}\left(f_{1}, \ldots, f_{n}\right)=g_{1}=f_{1} \sqcup \ldots \sqcup f_{n} .
$$

(ii) Let $\mathbf{w}=\left(w_{1}, \ldots, w_{n-1}, \widetilde{1}\right)$ with $w_{i} \sqsubseteq g_{n}$ for all $i \in\{1, \ldots, n-1\}$. Then $w_{i} \sqsubseteq g_{i}$ for all $i \in\{1, \ldots, n-1\}$, and we have

$$
\begin{aligned}
F_{\mathbf{w}}\left(f_{1}, \ldots, f_{n}\right) & =\left(w_{1} \sqcap g_{1}\right) \sqcup \ldots \sqcup\left(w_{n-1} \sqcap g_{n-1}\right) \sqcup\left(\widetilde{1} \sqcap g_{n}\right) \\
& =w_{1} \sqcup \ldots \sqcup w_{n-1} \sqcup g_{n}=g_{n}=f_{1} \sqcap \ldots \sqcap f_{n} .
\end{aligned}
$$

(iii) Let $\mathbf{w}=\left(\widetilde{0}, \ldots, \widetilde{0}, w_{k}=\widetilde{1}, \widetilde{0} \ldots, \widetilde{0}\right)$. Then

$$
\begin{aligned}
& F_{\mathbf{w}}\left(f_{1}, \ldots, f_{n}\right) \\
& =\left(w_{1} \sqcap g_{1}\right) \sqcup \ldots \sqcup\left(w_{k-1} \sqcap g_{k-1}\right) \sqcup\left(\widetilde{1} \sqcap g_{k}\right) \sqcup\left(w_{k+1} \sqcap g_{k+1}\right) \ldots \sqcup\left(w_{n} \sqcap g_{n}\right) \\
& =w_{1} \sqcup \ldots \sqcup w_{k-1} \sqcup g_{k} \sqcup\left(w_{k+1} \sqcap g_{k+1}\right) \ldots \sqcup\left(w_{n} \sqcap g_{n}\right)=g_{k} .
\end{aligned}
$$

Theorem 3.15. Let $\mathbf{w}=\left(w_{1}, \ldots, w_{n}\right)$ be a distributive weighting vector in $\mathcal{F}_{N C}$, and let $F_{\mathbf{w}}$ be an n-ary $O W A$ operator on $\mathcal{F}_{N C}$. Then:

(i) $F_{\mathbf{w}}$ is a symmetric operator, i.e.,

$$
F_{\mathbf{w}}\left(f_{1}, \ldots, f_{n}\right)=F_{\mathbf{w}}\left(f_{\sigma(1)}, \ldots, f_{\sigma(n)}\right)
$$

for all $f_{1}, \ldots, f_{n} \in \mathcal{F}_{N C}$, for each permutation $\sigma$ of $\{1, \ldots, n\}$. 
ON ALGEBRAIC PROPERTIES AND LINEARITY OF OWA OPERATORS...

(ii) $F_{\mathbf{w}}$ is an idempotent operator, i.e.,

Proof.

$$
F_{\mathbf{w}}(f, \ldots, f)=f \quad \text { for all } f \in \mathcal{F}_{N C} .
$$

(i) Immediately follows from Definition 3.8

(ii) According to Definition 3.5 we have

$$
\begin{aligned}
F_{\mathbf{w}}(f, \ldots, f) & =\left(w_{1} \sqcap f\right) \sqcup \ldots \sqcup\left(w_{n} \sqcap f\right) \\
& =\left(w_{1} \sqcup \ldots \sqcup w_{n}\right) \sqcap f=\widetilde{1} \sqcap f=f .
\end{aligned}
$$

We extend definitions of homogeneity and shift-invariance of aggregation functions from [0,1], see, e.g., [1], to fuzzy sets in [0,1].

Definition 3.16. An aggregation function $M: \mathcal{F}_{N C}^{n} \rightarrow \mathcal{F}_{N C}$ is homogeneous if for all $\lambda \in] 0, \infty\left[\right.$ and for all $\left(f_{1}, \ldots, f_{n}\right) \in \mathcal{F}_{N C}^{n}$ the following holds:

$$
M\left(\lambda f_{1}, \ldots, \lambda f_{n}\right)=\lambda M\left(f_{1}, \ldots, f_{n}\right)
$$

whenever $\left(\lambda f_{1}, \ldots, \lambda f_{n}\right) \in \mathcal{F}_{N C}^{n}$.

Definition 3.17. An aggregation function $M: \mathcal{F}_{N C}^{n} \rightarrow \mathcal{F}_{N C}$ is shift-invariant (or stable for translations) if for all $\lambda \in[0,1]$ and for all $\left(f_{1}, \ldots, f_{n}\right) \in \mathcal{F}_{N C}^{n}$ the following holds:

$$
M\left(f_{1}+\lambda, \ldots, f_{n}+\lambda\right)=M\left(f_{1}, \ldots, f_{n}\right)+\lambda
$$

whenever $\left(f_{1}+\lambda, \ldots, f_{n}+\lambda\right) \in \mathcal{F}_{N C}^{n}$.

Recall that for $f \in \mathcal{F}$ and appropriate $\lambda$ we have

$$
(\lambda f)(x)=f\left(\frac{x}{\lambda}\right) \quad \text { and } \quad(f+\lambda)(x)=f(x-\lambda),
$$

moreover, $\lambda f$ and $f+\lambda$ are normal convex fuzzy sets whenever $f$ is normal convex fuzzy set.

Theorem 3.18. OWA operator $F_{\mathbf{w}}: \mathcal{F}_{N C}^{n} \rightarrow \mathcal{F}_{N C}$ given by Definition 3.8 is not homogeneous.

Proof.

We give a counterexample. Let $\lambda=2, f_{1}=(0.3,0.4,0.5), f_{2}=(0.1,0.2,0.3)$, $w_{1}=(0.2,0.3,0.4)$ be triangular fuzzy sets in $[0,1], w_{2}=\widetilde{1}$ and $\mathbf{w}=\left(w_{1}, w_{2}\right)$. Then $\lambda f_{1}=(0.6,0.8,1), \lambda f_{2}=(0.2,0.4,0.6)$ and

$$
\begin{gathered}
F_{\mathbf{w}}\left(f_{1}, f_{2}\right)=\left(w_{1} \sqcap f_{1}\right) \sqcup\left(w_{2} \sqcap f_{2}\right)=w_{1} \sqcup f_{2}=w_{1}, \\
F_{\mathbf{w}}\left(\lambda f_{1}, \lambda f_{2}\right)=\left(w_{1} \sqcap \lambda f_{1}\right) \sqcup\left(w_{2} \sqcap \lambda f_{2}\right)=w_{1} \sqcup \lambda f_{2}=\lambda f_{2},
\end{gathered}
$$

hence $F_{\mathbf{w}}\left(\lambda f_{1}, \lambda f_{2}\right) \neq \lambda F_{\mathbf{w}}\left(f_{1}, f_{2}\right)$.

Although $F_{\mathbf{w}}$ is not homogeneous, the following properties of order relation $\sqsubseteq$ are satisfied. 
LEMma 3.19. Let $f_{1}, \ldots, f_{n} \in \mathcal{F}(R)$ and $\left.\lambda \in\right] 0, \infty[$. Then

(i) $\lambda f_{1} \sqcup \ldots \sqcup \lambda f_{n}=\lambda\left(f_{1} \sqcup \ldots \sqcup f_{n}\right)$,

(ii) $\lambda f_{1} \sqcap \ldots \sqcap \lambda f_{n}=\lambda\left(f_{1} \sqcap \ldots \sqcap f_{n}\right)$.

Proof.

(i) $\left(\lambda \mathrm{f}_{1} \sqcup \ldots \sqcup \lambda \mathrm{f}_{\mathrm{n}}\right)(\mathrm{z})=\sup _{x_{1} \vee \ldots \vee x_{n}=z}\left(\left(\lambda f_{1}\right)\left(x_{1}\right) \wedge \ldots \wedge\left(\lambda f_{n}\right)\left(x_{n}\right)\right)$

$$
\begin{aligned}
& =\sup _{\frac{x_{1}}{\lambda} \vee \ldots \vee \frac{x_{n}}{\lambda}=\frac{z}{\lambda}}\left(\left(f_{1}\right)\left(x_{1} / \lambda\right) \wedge \ldots \wedge\left(f_{n}\right)\left(x_{n} / \lambda\right)\right) \\
& =\left(f_{1} \sqcup \ldots \sqcup f_{n}\right)(z / \lambda)=\left(\lambda\left(f_{1} \sqcup \ldots \sqcup f_{n}\right)\right)(z) .
\end{aligned}
$$

(ii) The proof is analogous.

Corollary 3.20. Let $\lambda \in] 0, \infty\left[\right.$. If we take $\lambda f_{1}, \ldots, \lambda f_{n}$ instead of $f_{1}, \ldots, f_{n} \in$ $\mathcal{F}(R)$ in Lemma (3.4), then we get chain $\lambda g_{n} \sqsubseteq \ldots \sqsubseteq \lambda g_{1}$ instead of $g_{n} \sqsubseteq \ldots \sqsubseteq g_{1}$.

P r o of. Immediately follows from Lemma (3.4) and Lemma 3.19.

The similar results hold for shift-invariance of OWA operator $F_{\mathbf{w}}$.

Theorem 3.21. OWA operator $F_{\mathbf{w}}: \mathcal{F}_{N C}^{n} \rightarrow \mathcal{F}_{N C}$ given by Definition 3.8 is not shift-invariant.

Proof.

We give a counterexample. Let $\lambda=0.4, f_{1}=(0.3,0.4,0.5), f_{2}=(0.1,0.2,0.3)$, $w_{1}=(0.2,0.3,0.4)$ be triangular fuzzy sets in $[0,1], w_{2}=\widetilde{1}$ and $\mathbf{w}=\left(w_{1}, w_{2}\right)$. Then $f_{1}+\lambda=(0.7,0.8,0.9), f_{2}+\lambda=(0.5,0.6,0.7)$ and

$$
\begin{aligned}
F_{\mathbf{w}}\left(f_{1}, f_{2}\right) & =\left(w_{1} \sqcap f_{1}\right) \sqcup\left(w_{2} \sqcap f_{2}\right)=w_{1} \sqcup f_{2}=w_{1}, \\
F_{\mathbf{w}}\left(f_{1}+\lambda, f_{2}+\lambda\right) & =\left(w_{1} \sqcap\left(f_{1}+\lambda\right)\right) \sqcup\left(w_{2} \sqcap\left(f_{2}+\lambda\right)\right) \\
& =w_{1} \sqcup\left(f_{2}+\lambda\right)=f_{2}+\lambda,
\end{aligned}
$$

hence $F_{\mathbf{w}}\left(f_{1}+\lambda, f_{2}+\lambda\right) \neq F_{\mathbf{w}}\left(f_{1}, f_{2}\right)+\lambda$.

Although $F_{\mathrm{w}}$ is not shift-invariant, the following properties of order relation $\sqsubseteq$ are satisfied.

Lemma 3.22. Let $f_{1}, \ldots, f_{n} \in \mathcal{F}(R)$ and $\lambda \in R$. Then

(i) $\left(f_{1}+\lambda\right) \sqcup \ldots \sqcup\left(f_{n}+\lambda\right)=\left(f_{1} \sqcup \ldots \sqcup f_{n}\right)+\lambda$,

(ii) $\left(f_{1}+\lambda\right) \sqcap \ldots \sqcap\left(f_{n}+\lambda\right)=\left(f_{1} \sqcap \ldots \sqcap f_{n}\right)+\lambda$. 
ON ALGEBRAIC PROPERTIES AND LINEARITY OF OWA OPERATORS...

Proof.

(i)

$$
\begin{aligned}
& \left(\left(f_{1}+\lambda\right) \sqcup \ldots \sqcup\left(f_{n}+\lambda\right)\right)(z) \\
& =\sup _{x_{1} \vee \ldots \vee x_{n}=z}\left(\left(f_{1}+\lambda\right)\left(x_{1}\right) \wedge \ldots \wedge\left(f_{n}+\lambda\right)\left(x_{n}\right)\right) \\
& =\sup _{\left(x_{1}-\lambda\right) \vee \ldots \vee\left(x_{n}-\lambda\right)=z-\lambda}\left(\left(f_{1}\right)\left(x_{1}-\lambda\right) \wedge \ldots \wedge\left(f_{n}\right)\left(x_{n}-\lambda\right)\right) \\
& =\left(f_{1} \sqcup \ldots \sqcup f_{n}\right)(z-\lambda)=\left(\left(f_{1} \sqcup \ldots \sqcup f_{n}\right)+\lambda\right)(z) .
\end{aligned}
$$

(ii) The proof is analogous.

Corollary 3.23. Let $\lambda \in R$. If we take $f_{1}+\lambda, \ldots, f_{n}+\lambda$ instead of $f_{1}, \ldots, f_{n} \in$ $\mathcal{F}(R)$ in Lemma 3.4), then we get chain $g_{n}+\lambda \sqsubseteq \ldots \sqsubseteq g_{1}+\lambda$ instead of $g_{n} \sqsubseteq \ldots \sqsubseteq g_{1}$.

P r o of. Immediately follows from Lemma (3.4) and Lemma 3.22.

We show that OWA operator $F_{\mathbf{w}}$ given by Definition [3.8 is an extension of Yager's OWA operator on $[0,1]$, moreover, $F_{\mathbf{w}}$ is closed on the set of all closed subintervals of $[0,1]$. From now on we denote a singleton fuzzy set in $X$ given by some $a \in X$ as follows

$$
\widetilde{a}(x)= \begin{cases}1 & \text { if } x=a, \\ 0, & \text { otherwise }\end{cases}
$$

Theorem 3.24. Let $v_{1}, \ldots, v_{n}, a_{1}, \ldots, a_{n} \in[0,1], \mathbf{v}=\left(v_{1}, \ldots, v_{n}\right)$ and $\mathbf{w}=$ $\left(\widetilde{v_{1}}, \ldots, \widetilde{v_{n}}\right)$. Let $F_{\mathbf{w}}: \mathcal{F}_{N C}^{n} \rightarrow \mathcal{F}_{N C}$ be the corresponding OWA operator given by Definition 3.8 and $O W A_{\mathbf{v}}:[0,1]^{n} \rightarrow[0,1]$ be the standard Yager's OWA operator for real numbers given by Definition 2.1. Then

$$
F_{\mathbf{w}}\left(\widetilde{a_{1}}, \ldots, \widetilde{a_{n}}\right)=\widetilde{c}, \quad \text { where } \quad c=O W A_{\mathbf{v}}\left(a_{1}, \ldots, a_{n}\right),
$$

for all $v_{1}, \ldots, v_{n}$ such that $F_{\mathbf{w}}$ and $O W A_{\mathbf{v}}$ are defined.

Proof. Operators $F_{\mathbf{w}}$ and $O W A_{\mathbf{v}}$ are defined simultaneously if and only if the weighting vector $\left(\widetilde{v_{1}}, \ldots, \widetilde{v_{n}}\right)$ is distributive and $v_{1}+\cdots+v_{n}=1$, i.e., according to Corollary [3.7, $v_{k}=1$ for some $k \in\{1, \ldots, n\}$ and $v_{i}=0$ for all $i \in\{1, \ldots, n\}-\{k\}$. Then

$$
\begin{aligned}
& F_{\mathbf{w}}\left(\widetilde{a_{1}}, \ldots, \widetilde{a_{n}}\right) \\
& \left.\left.=\left(\widetilde{0} \sqcap g_{1}\right) \sqcup \ldots \sqcup \widetilde{0} \sqcap g_{k-1}\right) \sqcup\left(\widetilde{1} \sqcap g_{k}\right) \sqcup\left(\widetilde{0} \sqcap g_{k+1}\right) \sqcup \ldots \sqcup \widetilde{0} \sqcap g_{n}\right)=g_{k}=\widetilde{a_{\sigma(k)}}
\end{aligned}
$$

and

$$
\begin{aligned}
& O W A_{\mathbf{v}}\left(a_{1}, \ldots, a_{n}\right) \\
& =0 \cdot a_{\sigma(1)}+\cdots+0 \times a_{\sigma(k-1)}+1 \times a_{\sigma(k)}+0 \times a_{\sigma(k+1)}+\cdots+0 \times a_{\sigma(n)}=a_{\sigma(k)} .
\end{aligned}
$$

Recall that $g_{i}=\widetilde{a_{\sigma(i)}}$, for all $i \in\{1, \ldots, n\}$. 


\section{ZDENKO TAKÁČ}

From now on, $I([0,1])$ denotes the set of all closed subintervals of $[0,1]$, i.e.,

$$
I([0,1])=\{[a, b] \mid 0 \leq a \leq b \leq 1\},
$$

$\widetilde{a, b]}$ stands for the characteristic function of $[a, b]$, i.e.,

$$
\widetilde{[a, b]}(x)= \begin{cases}1, & \text { if } x \in[a, b], \\ 0, & \text { otherwise }\end{cases}
$$

and $K$ stands for the set of all characteristic functions of the closed subintervals of $[0,1]$. Recall that $(K, \sqcap, \sqcup, \sqsubseteq, \widetilde{0}, \widetilde{1})$ is a subalgebra of $\left(\mathcal{F}_{N C}, \sqcap, \sqcup, \sqsubseteq, \widetilde{0}, \widetilde{1}\right)$, see 13 .

TheOREM 3.25. Let $\mathbf{w}=\left(w_{1}, \ldots, w_{n}\right)$ be a distributive weighting vector in $(K, \sqcap, \sqcup, \sqsubseteq, \widetilde{0}, \widetilde{1})$ and $F_{\mathbf{w}}$ the corresponding OWA operator given by Definition 3.8 . If $f_{1}, \ldots, f_{n} \in K$, then $F_{\mathbf{w}}\left(f_{1}, \ldots, f_{n}\right) \in K$.

Pr o of. The proof is straightforward.

\section{Conclusion}

In [6], 8] an OWA operator on any complete lattice endowed with a t-norm and a t-conorm was introduced. In this paper we focused on OWA operators on one particular case of complete lattice, namely that of all normal convex fuzzy sets in $[0,1]$. We showed that the proposed OWA operator for fuzzy sets is an extension of standard OWA operator for real numbers and it possesses similar algebraic properties as standard one, such as boundary conditions, monotonicity, symmetry, idempotency, boundary from above by maximum and from below by minimum. However, the proposed operator is neither homogeneous nor shiftinvariant, i.e., it is not linear in contrast to the standard OWA operator.

We have restricted our attention on operations $\sqcap$ and $\sqcup$. Our next intention is to apply some other t-norms and t-conorms on the set of fuzzy truth values and study properties of corresponding OWA operators. Another line of our investigation is a relationship of the proposed OWA operators to existing operators, namely type-1 OWA operators [17], 18] and OWA operators for gradual intervals [12.

\section{REFERENCES}

[1] BeliakOV, G.-BUStince, H.-CAlvO, T.: A Practical Guide to Averaging Functions, in: Stud. Fuzziness Soft Comput., Vol. 329, Springer, 2016.

[2] BUSTINCE, H.-GALAR, M.-BEDREGAL, B.-KOLESÁROVÁ, A.-MESIAR, R.: A new approach to interval-valued choquet integrals and the problem of ordering in interval-valued fuzzy set applications, IEEE Trans. Fuzzy Syst. 21 (2013), 1150-1162. 


\section{ON ALGEBRAIC PROPERTIES AND LINEARITY OF OWA OPERATORS...}

[3] HARDinG, J.-WALKER, C.-WALKER, E.: Lattices of convex normal functions, Fuzzy Sets and Systems 159 (2008), 1061-1071.

[4] KACPRZYK, J.-ZADROŻNY, S.: Queries with fuzzy linguistic quantifiers for data of variable quality using some extended OWA operators, in: Adv. Intell. Syst. Comput., Vol. 400, Springer, 2016, pp. 295-305.

[5] KARNIK, N. N.-MENDEL, J. M.: Operations on type-2 fuzzy sets, Fuzzy Sets and Systems 122 (2000), 327-348.

[6] LIZASOAIN, I.-MORENO, C.: OWA operators defined on complete lattices, Fuzzy Sets and Systems 224 (2013), 36-52.

[7] MIZUMOTO, M.-TANAKA, K.: Some properties of fuzzy sets of type 2, Inform. Control 31 (1976), 312-340.

[8] OCHOA, G.-LIZASOAIn, I.-PATERnAin, D.-BUSTINCE, H.-PAL, N. R.: Some properties of lattice OWA operators and their importance in image processing, in: Proc. of the 16th World Congress of the Internat. Syst. Assoc.-IFSA '15 and the 9th Conf. of the European Society for Fuzzy Logic and Technology-EUSFLAT '15 (J. M. Alonso et al., eds.), Gijón, Spain, 2015, Atlantis Press, Amsterdam, 2015, pp. 1261-1265.

[9] STARCZEWSKI, J. T.: Extended triangular norms, Inform. Sci. 179 (2009), 742-757.

[10] TAKÁČ, Z.: Aggregation of fuzzy truth values, Inform. Sci. 271 (2014), 1-13.

[11] TAKÁČ, Z.: Type-2 aggregation operators, in: Proc. of the 8th Conf. of the European Society for Fuzzy Logic and Technol.-EUSFLAT '13 (G. Pasi et al., eds.), Milan, Italy, 2013, Atlantis Press, Amsterdam, pp. 165-170.

[12] TAKÁČ, Z.: A linear order and OWA operator for discrete gradual real numbers, in: Proc. of the 2015 Conf. of the Internat. Fuzzy Syst. Assoc. and the European Soc. for Fuzzy Logic and Technol.-IFSA-EUSFLAT '15 (J. M. Alonso et al., eds.), Gijón, Spain, 2015, Atlantis Press, Amsterdam, 2015. pp. 260-266.

[13] WALKER, C.-WALKER, E.: The algebra of fuzzy truth values, Fuzzy Sets and Systems 149 (2005), 309-347.

[14] WALKER, C.-WALKER, E.: T-norms for type-2 fuzzy sets, in: Proc. of the 2006 IEEE Internat. Conf. on Fuzzy Systems, Vancouver, Canada, 2006, IEEE, 2006, pp. 16-21.

[15] XU, Z. S.-DA, Q. L.: The uncertain OWA operator, Int. J. Intell. Syst. 17 (2002), $569-575$.

[16] YAGER, R. R.: On ordered weighted averaging aggregation operators in multicriteria decisionmaking, IEEE Trans. Syst. Man Cybern. 18 (1988), 183-190.

[17] ZHOU, S. M.-CHICLANA, F.-JOHN, R. I.-GARIBALDI, J. M.: Type-1 OWA operators for aggregating uncertain information with uncertain weights induced by type-2 linguistic quantifiers, Fuzzy Sets and Systems 159 (2008), 3281-3296.

[18] ZHOU, S. M.-CHICLANA, F.-JOHN, R. I.-GARIBALDI, J. M.: Alpha-level aggregation: A practical approach to type-1 OWA operation for aggregating uncertain information with applications to breast cancer treatments, IEEE Trans. Knowl. Data Eng. 23 (2011), $1455-1468$.

Institute of Information Engineering

Automation and Mathematics

Faculty of Chemical an Food Technology

Slovak University of Technology in Bratislava

Radlinského 9

SK-812-37 Bratislava

SLOVAKIA

E-mail: zdenko.takac@stuba.sk 